\title{
Nanotechnology Approaches in Tackling Cardiovascular Diseases
}

\author{
Ray Putra Prajnamitra, Hung-Chih Chen, Chen-Ju Lin, Li-Lun Chen and Patrick Ching-Ho Hsieh *
}

Institute of Biomedical Sciences, Academia Sinica, 128 Section 2 Academia Road, Nangang District, Taipei 115, Taiwan; ray.prajnamitra@ibms.sinica.edu.tw (R.P.P.); byronbio@ibms.sinica.edu.tw (H.-C.C.); cjlin@ibms.sinica.edu.tw (C.-J.L.); lilun0817@ibms.sinica.edu.tw (L.-L.C.)

* Correspondence: phsieh@ibms.sinica.edu.tw; Tel.: +886-2-2789-9170

Academic Editors: Iwona Cicha, László Dézsi and May Azzawi

Received: 26 April 2019; Accepted: 25 May 2019; Published: 27 May 2019

check for updates

\begin{abstract}
Cardiovascular diseases have continued to remain a leading cause of mortality and morbidity worldwide. Poor proliferation capability of adult cardiomyocytes disables the heart from regenerating new myocardium after a myocardial ischaemia event and therefore weakens the heart in the long term, which may result in heart failure and death. Delivery of cardioprotective therapeutics soon after the event can help to protect the heart from further cell death and improve cardiac function, but delivery methods and potential side effects of these therapeutics may be an issue. Advances in nanotechnology, particularly nanoparticles for drug delivery, have enabled researchers to obtain better drug targeting capability, thus increasing the therapeutic outcome. Detailed study of nanoparticles in vivo is useful as it can provide insight for future treatments. Nanogel can help to create a more favourable environment, not only for a sustained delivery of therapeutics, but also for a better navigation of the therapeutics to the targeted sites. Finally, if the damage to the myocardium is too severe for drug treatment, nanopatch can help to improve cardiac function and healing by becoming a platform for pluripotent stem cell-derived cardiomyocytes to grow for the purpose of cell-based regenerative therapy.
\end{abstract}

Keywords: cardiovascular diseases; drug delivery; nanoparticle; biodistribution; nanogel; nanopatch; nanomaterial; cell sheet

\section{Introduction}

Despite the rapid growth of the global healthcare industry in recent years, cardiovascular diseases (CVDs) have continued to be a prominent cause of mortality and morbidity. Earlier this year the American Heart Association reported that in 2016, CVDs contributed to around 17.6 million deaths worldwide [1]. Among the different types of diseases that fall under the umbrella of CVDs, ischaemic heart disease remains the most common cause of death.

Ischaemia is a condition where an occlusion takes place in the artery, interrupting blood flow and oxygen supply, causing damage to a specific area or tissue in the body. The occlusion can be caused by a clot due to atherosclerosis, thrombosis, or embolism. An ischaemic event that takes place in the heart deprives it of its blood and oxygen supply, resulting in myocardial infarction (MI) or heart attack. When this happens, the most common treatment is to restore the blood flow by relieving the occlusion as soon as possible. Paradoxically, however, the concomitant restoration of blood flow-also known as reperfusion-may inflict additional injury and induce further cell death through the formation of reactive oxygen species. Altogether, this phenomenon is known as the ischaemia/reperfusion (I/R) injury [2].

A heart that suffers from MI may lose around $25 \%$ of its cardiomyocytes (CMs) during the episode [3]. Poor proliferation capability of adult CMs renders the heart incapable of regenerating new 
CMs to replace those it has lost. As a result, it heals itself through the formation of fibrotic scar tissue which, unlike CMs, is not capable of beating. This healing process, known as ventricular remodeling, consequently increases the burden that the heart needs to withstand and therefore, slowly weakens its muscles. The heart will then deteriorate by gradually losing its contractility and muscle strength and, in the long term, this may result in congestive heart failure and ultimately, death.

It is therefore obvious that CMs are vital in maintaining heart function. Cell therapy using CMs derived from embryonic stem cells (ESCs) or induced pluripotent stem cells (iPSCs) provides a unique avenue in the treatment of CVDs, whereby new CMs are regenerated and engrafted on to patients. The progress in stem cell treatment research, however, has not been without its difficulties. Problems such as immunogenicity, slow CM maturation, transplantation issues, and potential formation of teratomas to name a few [3], are actively being addressed by many research groups worldwide. If the damage to the myocardium is not too severe, it is possible for cardioprotective therapeutics to be administered to the patients as an alternative to stem cell treatment, in the hope of protecting CMs from further damage and death following heart injury.

The advent of nanotechnology has revolutionised the field of biomedicine through the development of biocompatible nanomaterials. These nanomaterials have been extensively exploited for various biomedical purposes, including the treatment of CVDs, and have resulted in improved therapeutic benefits overall. Several applications of these nanomaterials include nanoparticles for drug delivery, scaffolds for stem cell modulation, in vivo imaging agents, and platforms for tissue engineering, among others.

In this review, we report the advances in the field to which our lab and others have contributed. We categorise the types of nanomaterials based on the material that they comprise: Nanoparticles, nanogel, and nanopatch. We also show their applications as well as challenges that are hampering the progress, which future research should take into consideration. Altogether, we highlight the considerable role that nanotechnology has played in advancing the treatment of CVDs.

\section{Nanoparticles}

\subsection{Nanoparticles for the Delivery of Therapeutics}

A wide range of therapeutics are available for heart treatment following an ischaemic episode. To ensure the delivery of these therapeutics to the myocardium however, direct intramyocardial injection or intracoronary catheterisation may be required [4]. We previously reported functionalised poly(lactic-co-glycolic acid) (PLGA) nanoparticles carrying insulin-like growth factor (IGF)-1, delivered to the myocardium via intramyocardial injection, which successfully provided cardioprotection to the heart, post-MI in mice [5]. Even though intramyocardial injection showed promising results, it is a very invasive technique and risks inflicting further damage to the heart. On the other hand, intracoronary catheterisation may not be suitable for some therapeutics and may risk inducing embolisation [4]. All of these reasons make intravenous delivery a better choice as it is less invasive and less risky.

When therapeutics are administered through systemic intravenous injection, they will go along the circulatory system. Taking advantage of this phenomena, we reported a successful prevention of thrombus formation in an acute hind limb thromboembolism murine model, using heparin-conjugated carbon nanocapsules delivered by intravenous injection [6]. Carbon nanocapsules were opted as they are more biocompatible in comparison to other carbon-based nanomaterials and more suitable for intravenous delivery [6,7]. Heparin-conjugated carbon nanocapsules were shown to display better antithrombotic activity and extend the formation time of the thrombus longer, compared to heparin or carbon nanocapsules alone [6].

As therapeutics go along the circulatory system after intravenous injection, they are taken up by various organs, retained, and ultimately cleared. Non-functionalised therapeutics are not capable of targeting the heart specifically and will mostly end up in reticuloendothelial organs, such as the liver and spleen [4]. Therefore, it is now common to "protect" the therapeutics by encapsulating them inside 
nanoparticles. Upon encapsulation, these therapeutics may have a different retention profile compared to their free form, depending on the physicochemical characteristics of the carriers. Drug encapsulation in nanoparticles can be advantageous as it can increase the retention and circulatory half-lives of the drugs $[4,5,8]$. The surface of these nanoparticles can also be modified with various functional groups that enable active targeting of nanoparticles to certain sites, cells, or tissues. A common example of surface customisation is the attachment of polyethylene glycol (PEG), also known as PEGylation. Nanoparticles that are PEGylated have been reported to possess increased stability and circulatory half-life as well as reduced risk of aggregation [4,8,9]. Surface customisation can also be performed using peptides [10], proteins [11], aptamers [12,13], or small molecules [14,15], which may enable the active targeting of the nanoparticles.

Nanoparticles without active targeting moieties are still capable of reaching diseased sites by passive targeting. The so-called enhanced permeability and retention (EPR) effect has been implicated as the reason behind passive targeting, whereby the vasculature of diseased or injured sites gradually become more permeable, enabling drug-containing nanoparticles to permeate through (Figure 1A). The EPR effect has been utilised for tumour targeting because it is persistent for a long duration [16,17]. The production of vascular permeability factor [18] as well as several compounds that enhance permeability compounds, such as nitric oxide, peroxynitrite, and bradykinin [19], have been linked to the increase of permeability of the diseased vasculature. On the other hand, the absence of lymphatic vessels in tumours $[19,20]$ and the unidirectional nature of extravasation through this vasculature [21] are thought to be the main causes of nanoparticle retention and accumulation in tumour sites.

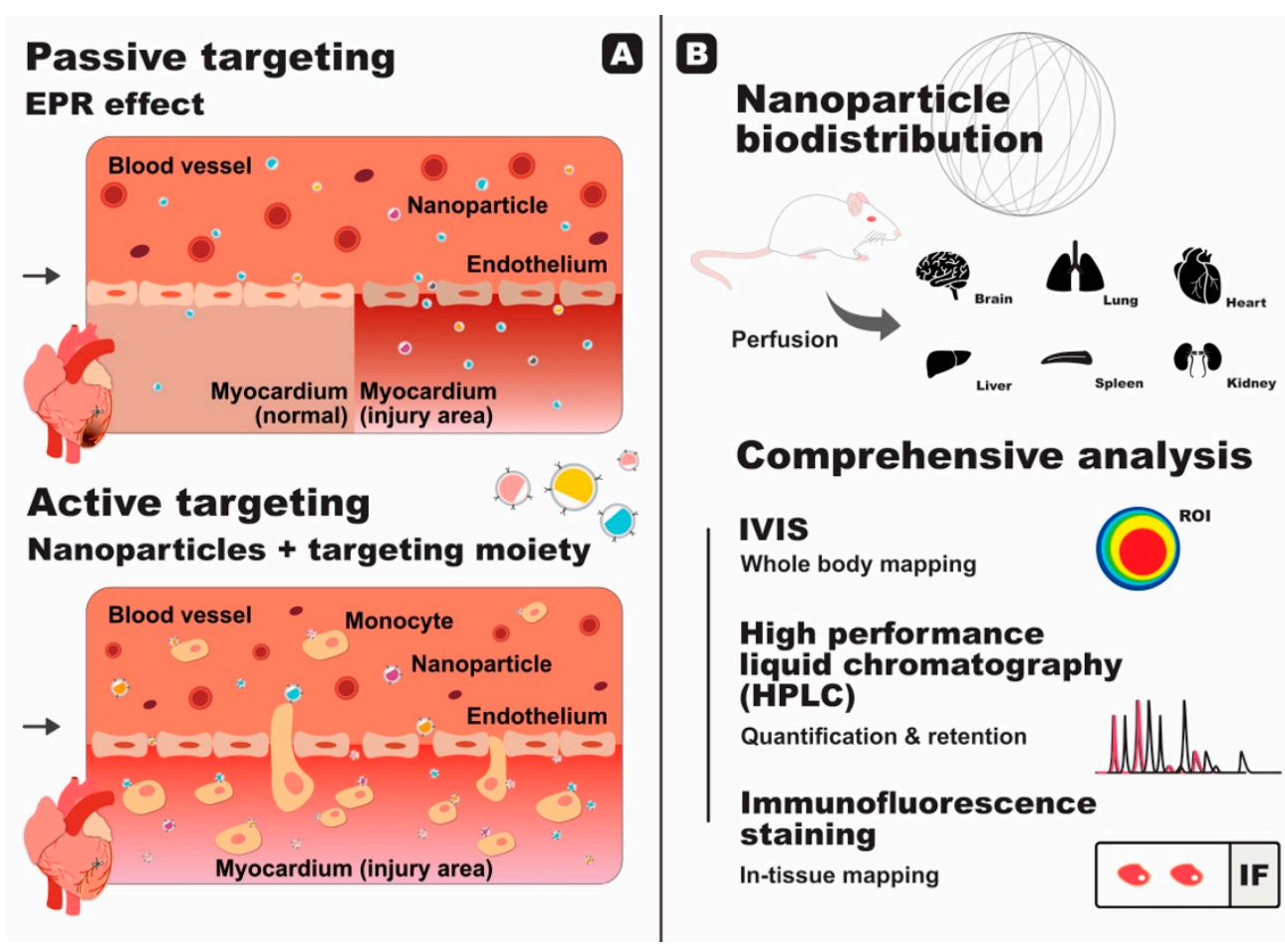

Figure 1. The use of functionalised nanoparticles for the treatment of cardiac ischaemic injury: (A) Passive targeting via the enhanced permeability and retention (EPR) effect in comparison to active targeting, using nanoparticles decorated with targeting moieties. Nanoparticles bearing monocyte-targeting moiety are able to actively attach onto the surface of monocytes and extravasate into the injured area; (B) Nanoparticles biodistribution study is indispensable in order to investigate the fate of nanoparticles in vivo. Perfusion is performed to rid the organs of freely circulating nanoparticles in the blood prior to organ collection. Overall, biodistribution study maps out the distribution of nanoparticles throughout the body and within the tissue. It also shows the amount retained in each tissue, providing researchers with a complete overview of the fate of their nanoparticles. 
The EPR effect in the myocardium after an ischaemic episode has also been reported [22]. The upregulation of vascular endothelial growth factor (VEGF) production takes place after MI to promote angiogenesis and restore oxygen supply to the damaged myocardium [23,24]. Furthermore, VEGF is also known to increase vascular permeability $[23,25,26]$, therefore resulting in the post-MI EPR effect in the myocardium [23]. Contrary to the EPR effect in tumours, however, post-MI EPR effect does not last long and begins to diminish after $24-48 \mathrm{~h}[11,22]$ with partial functional recovery observed after two weeks [22]. A long period of treatment is generally required to treat and heal the heart after an ischaemic episode to prevent negative left ventricular remodelling [27]. This time window is therefore too short for the delivery of the necessary therapeutics.

Despite this limitation, several studies have reported the use of the EPR effect for passive targeting of the heart after an ischaemic episode [27-30]. One of the studies reported the use of nanoparticles that are able to target the heart through the EPR effect. These nanoparticles self-aggregate upon stimulation by matrix metalloproteinase (MMPs) — enzymes that are present and upregulated in MI—in particular, MMP-2 and MMP-9. The aggregation forms a scaffold that is capable of retaining itself for up to 28 days post-MI [27]. Although the study has successfully increased the retention time, delivery of the nanoparticles would still have to be performed before the EPR effect diminishes. Thus, elimination of the reliance on the EPR effect is still the ideal solution. This can be achieved by exploiting nanoparticles decorated with active targeting moieties.

Scott et al. [31] made use of the increased expression of P-selectin within the vasculature that lies between normal and ischaemic myocardium by conjugating liposomes with an anti-P-selectin antibody. VEGF was encapsulated in these liposomes to promote angiogenesis in the infarct zone. The authors reported that delivery of the anti-P-selectin-conjugated liposomes to a rat MI model successfully improved vascularity and cardiac function, as indicated by the improvement of systolic function as well as the increase in fractional shortening [31].

In 2015, we reported a novel strategy to deliver therapeutics to the heart using liposome nanoparticles coated with a platelet membrane protein, which we coined platelet-like proteoliposome (PLP) [11]. Splenic monocytes are continuously recruited to the infarcted heart to aid with heart healing [32-34] in an EPR effect-independent manner (Figure 1A). It has also been reported that in MI patients, platelets are able to bind to circulating monocytes [35]. The PLPs were devised to take advantage of the platelet-monocyte binding, mimic this interaction, and use monocytes as a shuttle bus that can deliver the drugs to the heart. Cobalt protoporphyrin (CoPP) was chosen as the drug in this study. CoPP is able to suppress the inflammatory activity of macrophages by inducing heme oxygenase- 1 (HO-1) expression, but as a side effect, it is toxic to the liver and kidneys. CoPP-encapsulated PLPs successfully attached themselves to monocytes and piggybacked their way to the infarcted heart without relying on the EPR effect. Upon extravasation to the myocardium, the PLPs were phagocytised by macrophages and the ensuing release of CoPP directly inside the macrophages increased the therapeutic efficacy. Overall, CoPP-encapsulated PLPs displayed enhanced drug targeting capability and successful improvement in cardiac function with minimal side effects, compared to free CoPP or CoPP-encapsulated liposomes alone. Despite the significant increase, however, only around 5\% of the injected PLPs were detected in the heart, whilst the majority were trapped in the spleen and liver [11]. Even though this number is higher than what other research had reported, improvement should be made so that a higher number of nanoparticles are able to reach the heart and therefore increase the therapeutic outcome.

\subsection{Biodistribution Study of Nanoparticles In Vivo}

Research involving the study of nanoparticles in vivo usually includes a biodistribution study to investigate the fate of the nanoparticles in the body and to map out in which organs they are taken up. The biodistribution study is even more important when the nanoparticles contain active targeting moieties, in order to prove their targeting capability (Figure 1B).

Using fluorescent nanoparticles or nanoparticles conjugated to a fluorochrome, our lab has routinely used an in vivo imaging system (IVIS) to obtain biodistribution profiles under various 
physiological conditions $[4,36,37]$. The advantage of IVIS is that a whole-body analysis can be performed without sacrificing the animals, however, absolute quantification using IVIS is not recommended as signal saturation and tissue autofluorescence can make the quantification inaccurate [36]. Therefore, we routinely employ high performance liquid chromatography (HPLC) for absolute quantification purposes, due to its superior sensitivity and, more importantly, because it also involves extraction and separation of the fluorescent dye from the tissue. We also make use of immunofluorescence staining in order to further confirm and analyse the distribution within the cells or tissues [4,36,37].

Biodistribution studies are not limited to using fluorescent-based techniques as radiolabeled nanoparticles can be and have been used to assess biodistribution profiles [8,38]. Lameijer et al. [38] reported nanoparticles constructed from high density lipoprotein (HDL) human apolipoprotein A-I that encapsulated a small molecule inhibitor for tumour necrosis factor receptor-associated factor 6 (TRAF6). This treatment managed to rapidly reduce plaque inflammation in transgenic apolipoprotein E-deficient $\left(\right.$ Apoe $\left.^{-/-}\right)$atherosclerotic mice within one week of therapy. The biodistribution study in this research was carried out in murine and non-human primate models, using positron-emission tomography with magnetic resonance imaging (PET/MRI) [38].

Table 1. Size-dependent effect of nanoparticle biodistribution in mice, post-ischaemia/reperfusion (I/R) injury.

\begin{tabular}{|c|c|c|}
\hline $\begin{array}{l}\text { Nanoparticle } \\
\text { Size (nm) }\end{array}$ & $\begin{array}{l}\text { HPLC Quantification } \\
\text { (I/R vs. Sham) }\end{array}$ & $\begin{array}{c}\text { Observation through } \\
\text { Immunofluorescence Staining }\end{array}$ \\
\hline 20 & $\begin{array}{c}\text { Significantly higher in } \\
\text { I/R-injured hearts }(p \leq 0.05)\end{array}$ & $\begin{array}{l}\text { Sham hearts } \\
\text { Sparse, mostly outside blood vessels } \\
\text { I/R hearts } \\
\text { Accumulation in the injured left ventricle } \\
\text { Few in the uninjured right ventricle }\end{array}$ \\
\hline 100 & $\begin{array}{c}\text { Significantly higher in } \\
\text { I/R-injured hearts }(p \leq 0.05)\end{array}$ & $\begin{array}{c}\frac{\text { Sham hearts }}{\text { Very sparse }} \\
\text { I/R hearts } \\
\text { Clear localisation in the injured } \\
\text { left ventricle both inside and outside blood vessels } \\
\text { Almost none in the uninjured right ventricle }\end{array}$ \\
\hline 200 & $\begin{array}{c}\text { Significantly higher in } \\
\text { I/R-injured hearts }(p \leq 0.05)\end{array}$ & $\begin{array}{c}\text { Sham hearts } \\
\text { Sparse, mostly inside blood vessels } \\
\text { I/R hearts } \\
\text { Accumulation in the injured left } \\
\text { ventricle mostly inside blood vessels } \\
\text { Occasionally found in the uninjured right ventricle }\end{array}$ \\
\hline 500 & $\begin{array}{c}\text { Significantly higher in } \\
\text { I/R-injured hearts }(p \leq 0.05)\end{array}$ & $\begin{array}{l}\text { Sham hearts } \\
\text { Distributed all over the left and right ventricles } \\
\text { Colocalisation in the injured area with the } \\
\text { majority of nanoparticles within the blood vessels }\end{array}$ \\
\hline 1000 & $\begin{array}{l}\text { Higher in I/R-injured hearts } \\
\text { but not significant }(p=0.08)\end{array}$ & $\begin{array}{l}\text { Sham hearts } \\
\text { Found throughout the entire tissue } \\
\text { I/R hearts } \\
\text { Found throughout the entire tissue } \\
\text { Colocalisation observed in the injured left ventricle } \\
\text { Consistently found within blood vessels }\end{array}$ \\
\hline 2000 & $\begin{array}{l}\text { Higher in I/R-injured hearts } \\
\text { but not significant }(p=0.24)\end{array}$ & $\begin{array}{l}\text { Sham hearts } \\
\text { Found throughout the entire tissue } \\
\text { Found throughout the entire tissue } \\
\text { Colocalistion observed in the injured left ventricle } \\
\text { Large clusters found entrapped within blood vessels }\end{array}$ \\
\hline
\end{tabular}


To further highlight the importance of biodistribution studies, we previously reported the size-dependent effect of nanoparticle distribution in mice post-I/R injury. Various sizes of PEGylated polystyrene nanoparticles-with core diameters of 20, 100, 200, 500, 1000, and $2000 \mathrm{~nm}$-without active targeting moiety were administered via intravenous injection. Through HPLC, we found that the retention of 20,100,200, and $500 \mathrm{~nm}$ nanoparticles were significantly higher in I/R-injured hearts compared to sham hearts and no significant difference was found for the retention of 1000 and $2000 \mathrm{~nm}$ nanoparticles. Interestingly, immunofluorescence staining gave a more detailed insight into the distribution in the tissue level (Table 1), where 200 and $500 \mathrm{~nm}$ nanoparticles appeared to be entrapped in the blood vessel, indicative of the inability to extravasate into the myocardium due to their size [4]. The staining results also showed that there was a preferential accumulation of nanoparticles in the left ventricle (infarct area) compared to the right ventricle (healthy area), thereby underlining the role of the EPR effect in passive targeting. These findings suggest that the diameter of nanoparticles should ideally be kept below $100 \mathrm{~nm}$ in order to maximise the efficacy of passive targeting of I/R-injured hearts [4].

\section{Nanogel}

Protein and cell therapies have been considered as promising approaches for treating cardiovascular diseases. They are designed to cure the disease with direct tissue repair and regeneration, while interventions currently adopted only prevent further damage to the injured site. Clinical trials involving protein or cell therapy for the treatment of cardiovascular diseases, however, have failed to outperform the existing therapies. This is mainly caused by the poor retention of the active elements in the injured site. To solve this problem, nanomaterials, such as self-assembling peptide nanofibers [39], hyaluronic acid hydrogels [40], and alginate hydrogels [41], have been developed to serve as scaffolds for capturing therapeutics and maintaining an environment that favours cardiac repair.

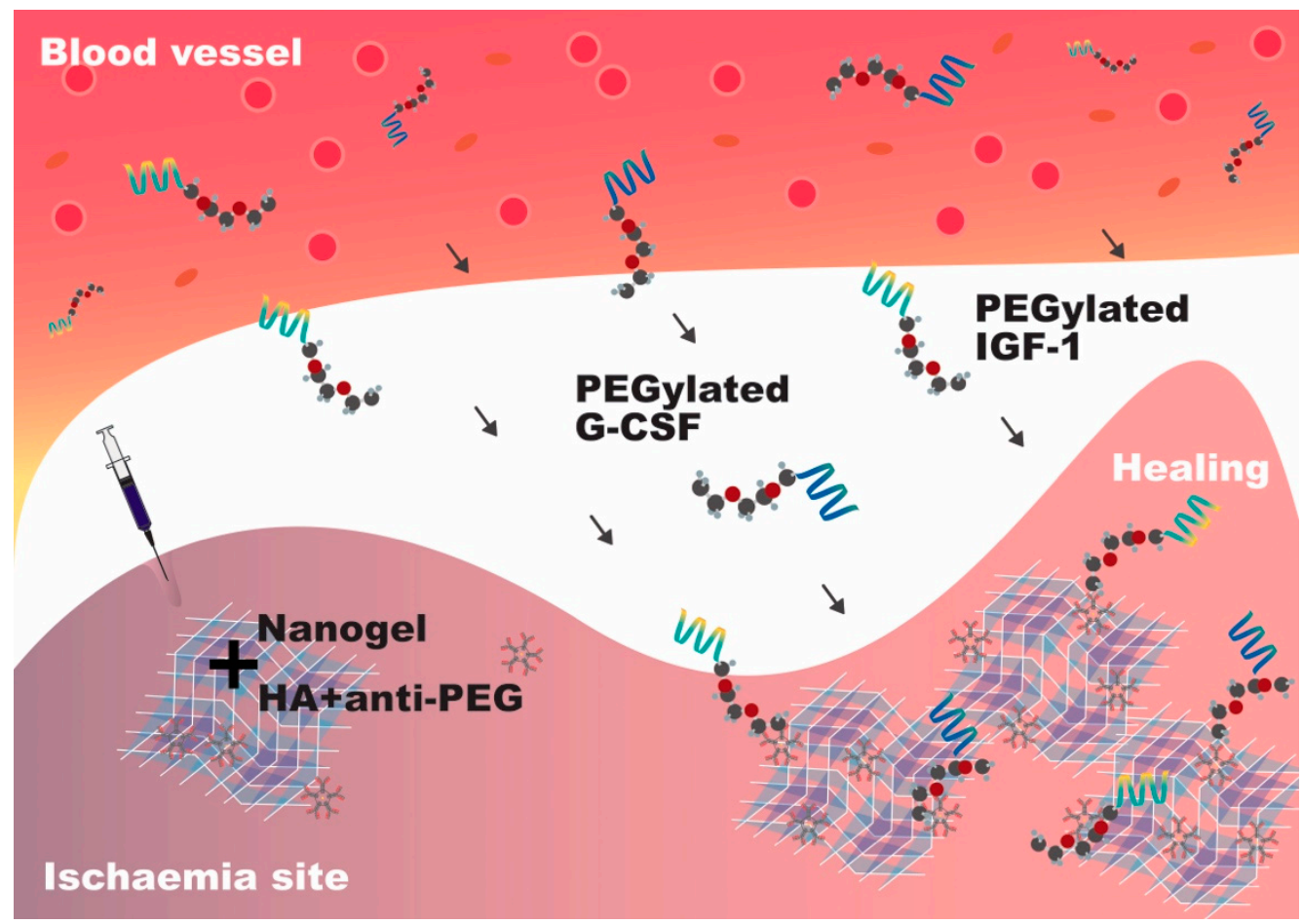

Figure 2. Reloadable multidrug capturing system. The nanogel containing anti-polyethylene glycol (PEG) antibody forms a scaffold which is capable of capturing multiple PEGylated factors from the blood vessel. PEGylated insulin-like growth factor (IGF)-1 is captured and retained, which reduces muscle cell death. Even after capturing the first factor, the reloadable scaffold is still capable of capturing and retaining the second factor, which is PEGylated granulocyte colony-stimulating factor (G-CSF), thereby promoting angiogenesis. 
In 2016, our group reported the development of a reloadable multidrug capturing system with anti-polyethylene glycol (PEG) antibody-containing hyaluronic acid (HA) hydrogels as a scaffold to capture PEGylated drugs (Figure 2). Using murine and porcine hind limb ischaemia models, we injected the anti-PEG-HA gel into the ischemic area, where it forms a network capable of capturing and retaining PEGylated IGF-1 and granulocyte colony-stimulating factor (G-CSF) that were intravenously injected successively. Treatment using both factors successfully reduced muscle cell death and promoted angiogenesis, respectively [40].

Using another disease model, we reported the use of a mixture of self-assembling and degradable peptide nanofibers and autologous bone marrow mononuclear cells to treat pigs with induced MI and found the treatment to be efficacious in enhancing both systolic and diastolic functions after injury [39]. We also proved that a mixture of nanofibers and vascular endothelial growth factors promotes arteriogenesis, which results in cardiac systolic function improvement and infarct size reduction within four weeks after MI in both murine and porcine models [42]. The biodegradable peptide nanofibers sustained the damaged site for three months and provided an advantageous microenvironment for cardiac repair as well as mechanical strength. It should be noted that the efficacy of cell therapy is time-dependent, as the treatment should be adopted within four days post-MI [43]. In summary, these studies demonstrated that nanofibers and nanogels are crucial in enhancing the efficacy of cell/protein therapy and restoring heart function in the long term.

\section{Nanopatch}

If the damage to the myocardium is very severe, only relying on drug treatment may not be sufficient and therefore transplantation or stem cell therapy can be opted. ESC- and iPSC-derived CMs are promising cell sources to repair the injured heart after MI. Direct injection of murine ESCs or human iPSCs (hiPSCs) to post-MI rat hearts has been shown to improve heart functions, however, long-term retention, survival, and integration of the implanted CMs are some of the major issues that need to be addressed $[44,45]$. To overcome these issues, bioengineered cardiac grafts have been developed to aid with post-MI heart repair.

Sekine et al. $[46,47]$ cultured neonatal rat CMs on their own or along with endothelial cells in temperature-responsive culture dishes to generate three-dimensional cell sheets. They transplanted the cell sheets into the infarct heart of a rat MI model and demonstrated improved long-term retention and survival of the transplanted CMs in the infarct area [46,47]. The left ventricular dysfunction can be assessed by decreased left ventricle wall thickness and fractional shortening, which represents the percentage change in the left ventricular cavity during systolic contraction. As a result, the hearts receiving cell sheet transplantation had improved fractional shortening and left ventricle wall thickness, as well as neovascularisation [46]. Using the same temperature-responsive cell sheet technology, Kawamura et al. [48] prepared hiPSC-derived CM sheets, combined with the omental flap, and transplanted the cell sheets into a porcine ischemic cardiomyopathy model. The omental flap was derived from the peritoneal layers and served as a blood supply for the cell sheets. Similarly, they demonstrated that the transplanted hiPSC-derived CMs displayed a better long-term survival on the cell sheets and significantly improved cardiac functions, such as the left ventricle ejection fraction and end diastolic and systolic volumes, in the post-MI porcine heart [48].

Apart from using cells alone, we developed an aligned-orientated nanofibrous electrospun patch that serves as a three-dimensional scaffold to align the injected rat neonatal CMs and endothelial cells [49]. Electrospinning techniques allow us to generate polyacrylonitrile nanofibers of $320 \pm 32$ $\mathrm{nm}$ in diameter and align the nanofibers into patches with a variation in fibre orientation of less than 10 degrees. Unlike the cell sheets alone, the nanofibrous patch itself is capable of providing the injured heart with some mechanical support. Moreover, rats with the cell-seeded cardiac patch implantation at two months post-MI displayed reductions in infarct size and fibrosis, and improvement in cardiac function and cardiac hemodynamic parameters. It is worth noting that the implanted cardiac patch 
formed a functional vascular connection with the host circulatory system, indicative of an improved blood supply to the cardiac patch and thus potential long-term survival of the patch (Figure 3).

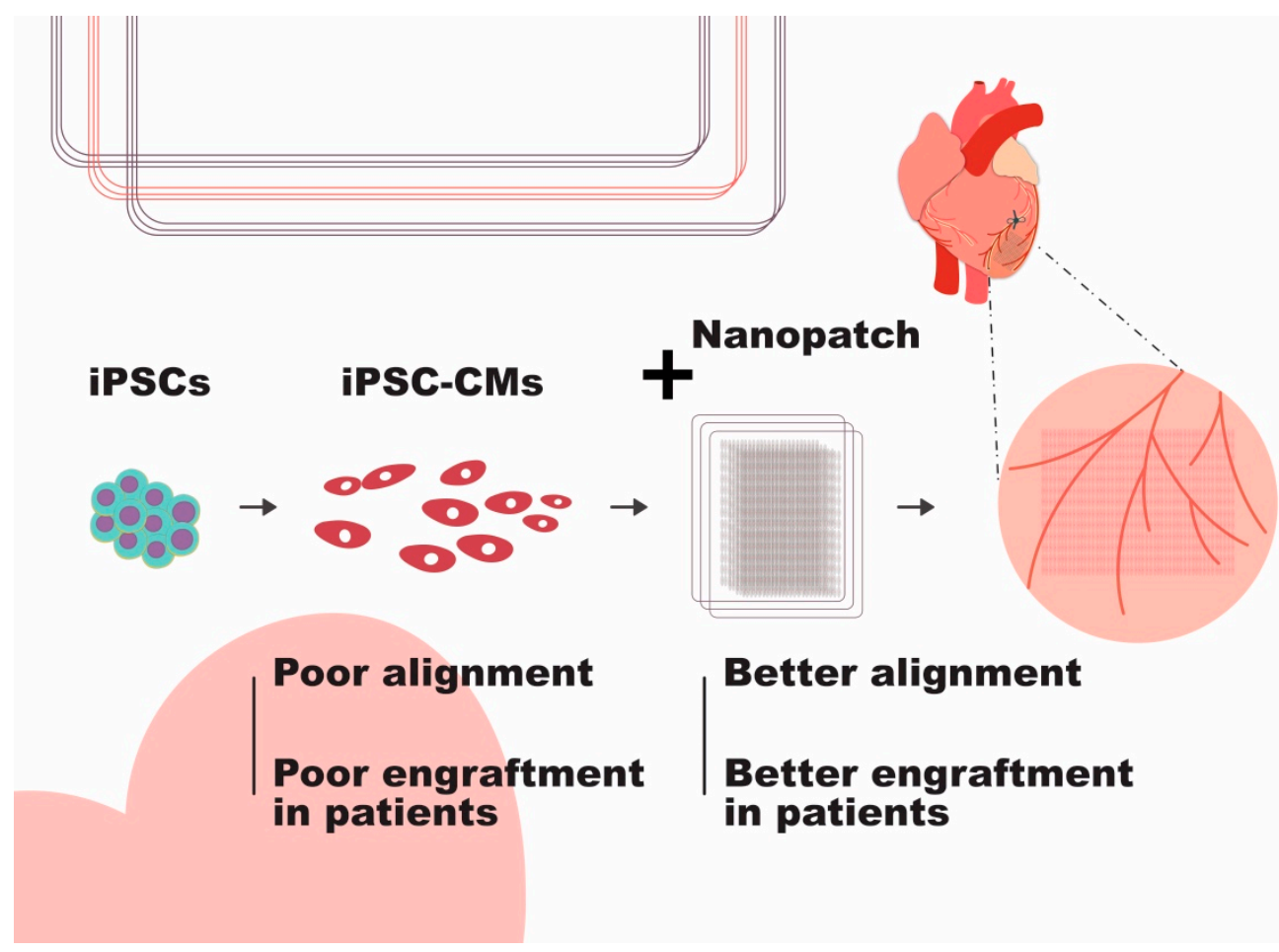

Figure 3. Engraftment of pluripotent stem cell-derived cardiomyocytes can be improved by attachment to a cell sheet. On their own, poor alignment and engraftment are the main issues. However, attaching them to a nanopatch results in better cardiomyocyte alignment and, ultimately, better engraftment in patients.

Before we can apply the cardiac patch technology for post-MI therapy, however, there are still some obstacles that needed to be overcome. It is important that the cardiac patch is functionally integrated onto the host heart, including vasculature connection to support the implanted cells for long-term survival. The electromechanical coupling of the implanted cardiac patch with the host heart is another issue that should be considered to prevent arrhythmia after transplantation.

\section{Conclusion and Future Developments}

The development of nanomaterials has greatly improved the therapeutic outcome for CVD treatments. Modifications of nanoparticles with active targeting moieties can greatly advance the delivery of cardioprotective drugs to the injured heart with reduced side effects, improving the therapeutic outcome overall. Further studies focusing on active targeting of nanoparticles instead of passive will help to achieve better therapeutics delivery. In addition, detailed biodistribution study helps to provide an insight that can enable better treatment in the future. The use of nanogel can help to create a more favourable environment, not only for sustained delivery of therapeutics, but also for a better navigation of the therapeutics to the target. Cardiac cell sheet provides a novel platform for cell-based therapy. Combined with nanofibrous materials, the cardiac patch can also provide mechanical support to the injured heart. A nanopatch capable of aiding electromechanical coupling of implanted CMs with the host heart would be a major breakthrough in stem cell therapy. Overall, despite the shortcomings, we have shown that nanotechnology plays a very important role in the treatment of CVDs, not only as a medium for drug delivery but also as a platform for cell-based therapy. We believe that nanotechnology, in particular, nanomedicine, is not only an alternative to stem cell treatment, but also a complement and its advancement will be very valuable for both fields. 
Funding: This work was supported by the Ministry of Science and Technology of Taiwan grants MOST 106-2811-B001-036, 106-2319-B-001-003 and 107-2314-B-004, the National Health Research Institutes grant EX106-10512SI and the Academia Sinica Program for Translational Innovation of Biopharmaceutical Development-Technology Supporting Platform Axis grant AS-KPQ-106-TSPA, the Thematic Research Program grant AS-107-TP-B12 and the Summit Research Program grant AS-SUMMIT-108.

Conflicts of Interest: The authors declare no conflict of interest.

\section{References}

1. Benjamin, E.J.; Muntner, P.; Alonso, A.; Bittencourt, M.S.; Callaway, C.W.; Carson, A.P.; Chamberlain, A.M.; Chang, A.R.; Cheng, S.; Das, S.R.; et al. Heart Disease and Stroke Statistics-2019 Update: A Report From the American Heart Association. Circulation 2019, 139, 56-528. [CrossRef]

2. Eltzschig, H.K.; Eckle, T. Ischemia and reperfusion-from mechanism to translation. Nat. Med. 2011, 17, $1391-1401$. [CrossRef] [PubMed]

3. Laflamme, M.A.; Murry, C.E. Heart regeneration. Nature 2011, 473, 326-335. [CrossRef]

4. Lundy, D.J.; Chen, K.-H.; Toh, E.K.W.; Hsieh, P.C.H. Distribution of Systemically Administered Nanoparticles Reveals a Size-Dependent Effect Immediately following Cardiac Ischaemia-Reperfusion Injury. Sci. Rep. 2016, 6, 25613. [CrossRef]

5. Chang, M.Y.; Yang, Y.J.; Chang, C.H.; Tang, A.C.L.; Liao, W.Y.; Cheng, F.Y.; Yeh, C.S.; Lai, J.J.; Stayton, P.S.; Hsieh, P.C.H. Functionalized nanoparticles provide early cardioprotection after acute myocardial infarction. J. Control. Release 2013, 170, 287-294. [CrossRef]

6. Tang, A.C.L.; Chang, M.-Y.; Tang, Z.C.W.; Li, H.-J.; Hwang, G.-L.; Hsieh, P.C.H. Treatment of Acute Thromboembolism in Mice Using Heparin-Conjugated Carbon Nanocapsules. ACS Nano 2012, 6, 6099-6107. [CrossRef]

7. Tang, A.C.L.; Hwang, G.-L.; Tsai, S.-J.; Chang, M.-Y.; Tang, Z.C.W.; Tsai, M.-D.; Luo, C.-Y.; Hoffman, A.S.; Hsieh, P.C.H. Biosafety of Non-Surface Modified Carbon Nanocapsules as a Potential Alternative to Carbon Nanotubes for Drug Delivery Purposes. PLoS ONE 2012, 7, e32893. [CrossRef]

8. Schipper, M.L.; Iyer, G.; Koh, A.L.; Cheng, Z.; Ebenstein, Y.; Aharoni, A.; Keren, S.; Bentolila, L.A.; Li, J.; Rao, J.; et al. Particle size, surface coating, and PEGylation influence the biodistribution of quantum dots in living mice. Small 2009, 5, 126-134. [CrossRef]

9. Murphy, A.J.; Funt, S.; Gorman, D.; Tall, A.R.; Wang, N. Pegylation of High-Density Lipoprotein Decreases Plasma Clearance and Enhances Antiatherogenic Activity. Circ. Res. 2013, 113, e1-e9. [CrossRef] [PubMed]

10. Santi, M.; Maccari, G.; Mereghetti, P.; Voliani, V.; Rocchiccioli, S.; Ucciferri, N.; Luin, S.; Signore, G. Rational Design of a Transferrin-Binding Peptide Sequence Tailored to Targeted Nanoparticle Internalization. Bioconjug. Chem. 2017, 28, 471-480. [CrossRef] [PubMed]

11. Cheng, B.; Toh, E.K.W.; Chen, K.H.; Chang, Y.C.; Hu, C.M.J.; Wu, H.C.; Chau, L.Y.; Chen, P.; Hsieh, P.C.H. Biomimicking Platelet-Monocyte Interactions as a Novel Targeting Strategy for Heart Healing. Adv. Healthc. Mater. 2016, 5, 2686-2697. [CrossRef] [PubMed]

12. Sun, H.; Zu, Y. Aptamers and their applications in nanomedicine. Small 2015, 11, 2352-2364. [CrossRef]

13. Belyanina, I.V.; Zamay, T.N.; Zamay, G.S.; Zamay, S.S.; Kolovskaya, O.S.; Ivanchenko, T.I.; Denisenko, V.V.; Kirichenko, A.K.; Glazyrin, Y.E.; Garanzha, I.V.; et al. In Vivo Cancer Cells Elimination Guided by AptamerFunctionalized Gold-Coated Magnetic Nanoparticles and Controlled with Low Frequency Alternating Magnetic Field. Theranostics 2017, 7, 3326-3337. [CrossRef]

14. Weissleder, R.; Kelly, K.; Sun, E.Y.; Shtatland, T.; Josephson, L. Cell-specific targeting of nanoparticles by multivalent attachment of small molecules. Nat. Biotechnol. 2005, 23, 1418-1423. [CrossRef]

15. Van Haute, D.; Liu, A.T.; Berlin, J.M. Coating Metal Nanoparticle Surfaces with Small Organic Molecules Can Reduce Nonspecific Cell Uptake. ACS Nano 2018, 12, 117-127. [CrossRef] [PubMed]

16. Maeda, H. Tumor-Selective Delivery of Macromolecular Drugs via the EPR Effect: Background and Future Prospects. Bioconjug. Chem. 2010, 21, 797-802. [CrossRef]

17. Prabhakar, U.; Maeda, H.; Jain, R.K.; Sevick-Muraca, E.M.; Zamboni, W.; Farokhzad, O.C.; Barry, S.T.; Gabizon, A.; Grodzinski, P.; Blakey, D.C. Challenges and Key Considerations of the Enhanced Permeability and Retention Effect for Nanomedicine Drug Delivery in Oncology. Cancer Res. 2013, 73, 2412-2417. [CrossRef]

18. Senger, D.; Galli, S.; Dvorak, A.; Perruzzi, C.; Harvey, V.; Dvorak, H. Tumor cells secrete a vascular permeability factor that promotes accumulation of ascites fluid. Science 1983, 219, 983-985. [CrossRef] 
19. Maeda, H.; Wu, J.; Sawa, T.; Matsumura, Y.; Hori, K. Tumor vascular permeability and the EPR effect in macromolecular therapeutics: A review. J. Control. Release 2000, 65, 271-284. [CrossRef]

20. Maeda, H. A New Concept for Macromolecular Therapeutics in Cancer Chemotherapy: Mechanism of Tumoritropic Accumulation of Proteins and the Antitumor Agent Smancs. Cancer Res. 1986, 46, 6387-6392.

21. Noguchi, Y.; Wu, J.; Duncan, R.; Strohalm, J.; Ulbrich, K.; Akaike, T.; Maeda, H. Early phase tumor accumulation of Macromolecules: A great difference in clearance rate between tumor and normal tissues. Jpn. J. Cancer Res. 1998, 89, 307-314. [CrossRef] [PubMed]

22. Horwitz, L.D.; Kaufman, D.; Keller, M.W.; Kong, Y. Time course of coronary endothelial healing after injury due to ischemia and reperfusion. Circulation 1994, 90, 2439-2447. [CrossRef]

23. Banai, S.; Shweiki, D.; Pinson, A.; Chandra, M.; Lazarovici, G.; Keshet, E. Upregulation of vascular endothelial growth factor expression induced by myocardial ischaemia: Implications for coronary angiogenesis. Cardiovasc. Res. 1994, 28, 1176-1179. [CrossRef]

24. Shweiki, D.; Itin, A.; Soffer, D.; Keshet, E. Vascular endothelial growth factor induced by hypoxia may mediate hypoxia-initiated angiogenesis. Nature 1992, 359, 843-845. [CrossRef] [PubMed]

25. Leung, D.; Cachianes, G.; Kuang, W.; Goeddel, D.; Ferrara, N. Vascular endothelial growth factor is a secreted angiogenic mitogen. Science 1989, 246, 1306-1309. [CrossRef]

26. Keck, P.; Hauser, S.; Krivi, G.; Sanzo, K.; Warren, T.; Feder, J.; Connolly, D. Vascular permeability factor, an endothelial cell mitogen related to PDGF. Science 1989, 246, 1309-1312. [CrossRef] [PubMed]

27. Nguyen, M.M.; Carlini, A.S.; Chien, M.P.; Sonnenberg, S.; Luo, C.; Braden, R.L.; Osborn, K.G.; Li, Y.; Gianneschi, N.C.; Christman, K.L. Enzyme-Responsive Nanoparticles for Targeted Accumulation and Prolonged Retention in Heart Tissue after Myocardial Infarction. Adv. Mater. 2015, 27, 5547-5552. [CrossRef] [PubMed]

28. Lukyanov, A.N.; Hartner, W.C.; Torchilin, V.P. Increased accumulation of PEG-PE micelles in the area of experimental myocardial infarction in rabbits. J. Control. Release 2004, 94, 187-193. [CrossRef] [PubMed]

29. Geelen, T.; Paulis, L.E.; Coolen, B.F.; Nicolay, K.; Strijkers, G.J. Passive targeting of lipid-based nanoparticles to mouse cardiac ischemia-reperfusion injury. Contrast Media Mol. Imaging 2013, 8, 117-126. [CrossRef] [PubMed]

30. Nakano, Y.; Matoba, T.; Tokutome, M.; Funamoto, D.; Katsuki, S.; Ikeda, G.; Nagaoka, K.; Ishikita, A.; Nakano, K.; Koga, J.; et al. Nanoparticle-Mediated Delivery of Irbesartan Induces Cardioprotection from Myocardial Ischemia-Reperfusion Injury by Antagonizing Monocyte-Mediated Inflammation. Sci. Rep. 2016, 6, 29601. [CrossRef]

31. Scott, R.C.; Rosano, J.M.; Ivanov, Z.; Wang, B.; Chong, P.L.-G.; Issekutz, A.C.; Crabbe, D.L.; Kiani, M.F. Targeting VEGF-encapsulated immunoliposomes to MI heart improves vascularity and cardiac function. FASEB J. 2009, 23, 3361-3367. [CrossRef]

32. Nahrendorf, M.; Swirski, F.K.; Aikawa, E.; Stangenberg, L.; Wurdinger, T.; Figueiredo, J.-L.; Libby, P.; Weissleder, R.; Pittet, M.J. The healing myocardium sequentially mobilizes two monocyte subsets with divergent and complementary functions. J. Exp. Med. 2007, 204, 3037-3047. [CrossRef]

33. Swirski, F.K.; Nahrendorf, M.; Etzrodt, M.; Wildgruber, M.; Cortez-Retamozo, V.; Panizzi, P.; Figueiredo, J.-L.; Kohler, R.H.; Chudnovskiy, A.; Waterman, P.; et al. Identification of Splenic Reservoir Monocytes and Their Deployment to Inflammatory Sites. Science 2009, 325, 612-616. [CrossRef]

34. Jung, K.; Kim, P.; Leuschner, F.; Gorbatov, R.; Kim, J.K.; Ueno, T.; Nahrendorf, M.; Yun, S.H. Endoscopic time-lapse imaging of immune cells in infarcted mouse hearts. Circ. Res. 2013, 112, 891-899. [CrossRef] [PubMed]

35. Alam, S.; Jha, A.; Laan, C.A.; Sarma, J.; Dransfield, I.; Fox, K.A.A. Increased Platelet Binding to Circulating Monocytes in Acute Coronary Syndromes. Circulation 2002, 105, 2166-2171. [CrossRef]

36. Liao, W.Y.; Li, H.J.; Chang, M.-Y.; Tang, A.C.L.; Hoffman, A.S.; Hsieh, P.C.H. Comprehensive characterizations of nanoparticle biodistribution following systemic injection in mice. Nanoscale 2013, 5, 11079-11086. [CrossRef]

37. Chen, K.H.; Lundy, D.J.; Toh, E.K.W.; Chen, C.H.; Shih, C.; Chen, P.; Chang, H.C.; Lai, J.J.; Stayton, P.S.; Hoffman, A.S.; et al. Nanoparticle distribution during systemic inflammation is size-dependent and organ-specific. Nanoscale 2015, 7, 15863-15872. [CrossRef]

38. Lameijer, M.; Binderup, T.; Van Leent, M.M.T.; Senders, M.L.; Fay, F.; Malkus, J.; Sanchez-Gaytan, B.L.; Teunissen, A.J.P.; Karakatsanis, N.; Robson, P.; et al. Efficacy and safety assessment of a TRAF6-targeted nanoimmunotherapy in atherosclerotic mice and non-human primates. Nat. Biomed. Eng. 2018, 2, $279-292$. [CrossRef] [PubMed] 
39. Lin, Y.D.; Yeh, M.L.; Yang, Y.J.; Tsai, D.C.; Chu, T.Y.; Shih, Y.Y.; Chang, M.Y.; Liu, Y.W.; Tang, A.C.L.; Chen, T.Y.; et al. Intramyocardial peptide nanofiber injection improves postinfarction ventricular remodeling and efficacy of bone marrow cell therapy in pigs. Circulation 2010, 122, S132-S141. [CrossRef]

40. Wu, J.P.J.; Cheng, B.; Roffler, S.R.; Lundy, D.J.; Yen, C.Y.T.; Chen, P.; Lai, J.J.; Pun, S.H.; Stayton, P.S.; Hsieh, P.C.H. Reloadable multidrug capturing delivery system for targeted ischemic disease treatment. Sci. Transl. Med. 2016, 8, 365ra160. [CrossRef]

41. Landa, N.; Miller, L.; Feinberg, M.S.; Holbova, R.; Shachar, M.; Freeman, I.; Cohen, S.; Leor, J. Effect of injectable alginate implant on cardiac remodeling and function after recent and old infarcts in rat. Circulation 2008, 117, 1388-1396. [CrossRef]

42. Lin, Y.-D.; Luo, C.-Y.; Hu, Y.-N.; Yeh, M.-L.; Hsueh, Y.-C.; Chang, M.-Y.; Tsai, D.-C.; Wang, J.-N.; Tang, M.-J.; Wei, E.I.H.; et al. Instructive nanofiber scaffolds with VEGF create a microenvironment for arteriogenesis and cardiac repair. Sci. Transl. Med. 2012, 4, 146ra109. [CrossRef] [PubMed]

43. Chang, M.-Y.; Chang, C.-H.; Chen, C.-H.; Cheng, B.; Lin, Y.-D.; Luo, C.-Y.; Wu, H.-L.; Yang, Y.-J.; Chen, J.-H.; Hsieh, P.C.H. The Time Window for Therapy with Peptide Nanofibers Combined with Autologous Bone Marrow Cells in Pigs after Acute Myocardial Infarction. PLoS ONE 2015, 10, e0115430. [CrossRef] [PubMed]

44. Chow, A.; Stuckey, D.J.; Kidher, E.; Rocco, M.; Jabbour, R.J.; Mansfield, C.A.; Darzi, A.; Harding, S.E.; Stevens, M.M.; Athanasiou, T. Human Induced Pluripotent Stem Cell-Derived Cardiomyocyte Encapsulating Bioactive Hydrogels Improve Rat Heart Function Post Myocardial Infarction. Stem Cell Reports 2017, 9 , 1415-1422. [CrossRef]

45. Min, J.-Y.; Yang, Y.; Sullivan, M.F.; Ke, Q.; Converso, K.L.; Chen, Y.; Morgan, J.P.; Xiao, Y.-F. Long-term improvement of cardiac function in rats after infarction by transplantation of embryonic stem cells. J. Thorac. Cardiovasc. Surg. 2003, 125, 361-369. [CrossRef] [PubMed]

46. Sekine, H.; Shimizu, T.; Dobashi, I.; Matsuura, K.; Hagiwara, N.; Takahashi, M.; Kobayashi, E.; Yamato, M.; Okano, T. Cardiac Cell Sheet Transplantation Improves Damaged Heart Function via Superior Cell Survival in Comparison with Dissociated Cell Injection. Tissue Eng. Part A 2011, 17, 2973-2980. [CrossRef]

47. Sekine, H.; Shimizu, T.; Hobo, K.; Sekiya, S.; Yang, J.; Yamato, M.; Kurosawa, H.; Kobayashi, E.; Okano, T. Endothelial Cell Coculture Within Tissue-Engineered Cardiomyocyte Sheets Enhances Neovascularization and Improves Cardiac Function of Ischemic Hearts. Circulation 2008, 118, S145-S152. [CrossRef] [PubMed]

48. Kawamura, M.; Miyagawa, S.; Fukushima, S.; Saito, A.; Miki, K.; Funakoshi, S.; Yoshida, Y.; Yamanaka, S.; Shimizu, T.; Okano, T.; et al. Enhanced Therapeutic Effects of Human iPS Cell Derived-Cardiomyocyte by Combined Cell-Sheets with Omental Flap Technique in Porcine Ischemic Cardiomyopathy Model. Sci. Rep. 2017, 7, 8824. [CrossRef]

49. Lin, Y.D.; Ko, M.C.; Wu, S.T.; Li, S.F.; Hu, J.F.; Lai, Y.J.; Harn, H.I.-C.; Laio, I.C.; Yeh, M.L.; Yeh, H.I.; et al. A nanopatterned cell-seeded cardiac patch prevents electro-uncoupling and improves the therapeutic efficacy of cardiac repair. Biomater. Sci. 2014, 2, 567-580. [CrossRef] [PubMed] 